\title{
Dynamical characteristics of wave-excited channel flow
}

\author{
S SELVARAJAN ${ }^{1}$ and V VASANTA RAM ${ }^{2}$ \\ ${ }^{1}$ National Aeronautical Laboratory, Vimanapura Road, Bangalore \\ 560017 , India \\ ${ }^{2}$ Institut für Thermo- und Fluiddynamik, Ruhr-Universität, Bochum, \\ Germany
}

\begin{abstract}
This paper is part of a study on the receptivity characteristics of the shear flow in a channel whose walls are subjected to a wave-like excitation. The small amplitude forced wavy wall motion is characterised by a wave number vector $\lambda_{1}, \lambda_{2}$ and a frequency $\omega_{g}$. The basic flow in the problem is a superposition of the Poiseuille flow and a periodic component that corresponds to the wave excitation of the wall. The aim of the study is to examine the susceptibility of this flow to transition. The problem is approached through studying the stability characteristics of the basic flow with respect to small disturbances. The theoretical framework for this purpose is Floquet theory. The solution procedure for solving the eigenvalue problem is the spectral collocation method. Preliminary results showing the influence of the amplitude and the wave number of the wall excitation on the stability boundary of the flow are presented.
\end{abstract}

Keywords. Dynamical characteristics; wave-excited channel flow; forced wavy wall motion; stability characteristics; spectral collocation method.

\section{Introduction}

In the broad area of study under the title "the stability of fluid motions", a subject that has attracted increased attention in recent years is the response to further disturbances of the shear flow that is excited by waves travelling in the plane of the walls. Besides the inherent fundamental interest that disturbance-propagation problems hold as such in fluid mechanics, the possibility of influencing transition and thus of managing turbulence through wall excitation has been recognised for some time now and this has lent additional impetus to research on this subject. It is therefore not surprising to find this subject occupying a prominent position in papers at conferences on turbulence control and management (see e.g. Liepmann \& Narasimha 1988), and at special sessions at larger conferences. Of the latter we mention here only the first European Fluid Mechanics Conference, 1991 (Cambridge, UK), The SIAM Conference on Applications of Dynamical Systems, 1992 (Salt Lake City), and the Fourth European Turbulence Conference 1992 (Delft, Netherlands).

In studies of disturbance propagation in these flows the points of main interest centre around the effects of the wall-wave excitation parameters, viz. its frequency, 
wave number (vector) and amplitude, on the growth (or decay) characteristics of the further disturbances. At moderate Reynolds numbers one is particularly interested in the shift in the surface of neutral stability due to the wave excitation at the wall since this is an indication of the susceptibility of the flow to transition to turbulence. When the Reynolds numbers are much larger, the propagation of disturbances has to be studied in a flow that is already turbulent and this belongs to the realm of "management" of turbulence through the wave excitation of the walls. The latter context requires for its understanding a much deeper insight into the turbulence mechanism in the flow than is available today. It poses a problem of formidable diversity and complexity. As against this, the former has the advantage of being more tractable, yet retaining some, if not all, physical elements that characterise a turbulent flow. It is therefore better suited for study at the present stage and therefore defines the scope of this work. The disturbance propagation problem in the laminar flow with wave excited walls, despite its conceptual simplicity, is a difficult one, not only because it involves a larger number of parameters than the classical problem with unexcited walls, but also due to additional physical phenomenon that are encountered due to the wave-like component present in the basic flow. These call for a modification of the mathematical tools used to handle these problems.

The subject of the present paper is the propagation of small amplitude disturbances in the channel flow whose walls undergo a travelling wave type of deformation of one wave number and frequency only. We also regard the amplitude of the wave excitation at the wall to be sufficiently small, so that the basic flow in which disturbance propagation is to be studied comprises only one wave component superposed upon the classical fully developed flow with a parabolic velocity profile in a channel with rigid walls. The number of parameters involved in this problem is much larger than in the classical problem and its thorough investigation calls for a more extensive study than is possible in the relatively brief time span of around two years over which this work has been in progress. Even with the limited scope set, the study is too extensive to be accommodated within this paper, so we restrict ourselves for the present primarily to outlining our approach and presenting a selection of preliminary results that we have obtained so far. The reader interested in further details is referred to Selvarajan \& Vasanta Ram (1991).

\section{The basic flow}

The equations of motion for an incompressible fluid arre, in the usual notation,

$$
\begin{aligned}
& \partial u_{1} / \partial x_{1}=0, \quad l=1,2,3 \\
& \frac{\partial u_{1}}{\partial t}+u_{m} \frac{\partial u_{1}}{\partial x_{m}}=-\frac{\partial p}{\partial x_{1}}+\frac{1}{\operatorname{Re}} \frac{\partial^{2} u_{1}}{\partial x_{m} \partial x_{m}}, \quad l, m=1,2,3,
\end{aligned}
$$

where the lengths are referred to the mean semi-channel height $H$, the velocity components to the mean centre line velocity $U_{0}$, the time to $H / U_{0}$, the pressure to $\rho U_{0}^{2}$ and the Reynolds number $\operatorname{Re}$ is based on $H$ and $U_{0}$.

We specify the wall motion through the following expression (see figure 1),

$$
y_{w}\left(t, x_{1}, x_{3}\right)= \pm 1 \mp \varepsilon_{w} \operatorname{Real}\left(\exp \left[i\left(\lambda_{1} x_{1}+\lambda_{3} x_{3}-\omega_{g} t\right)\right]\right),
$$




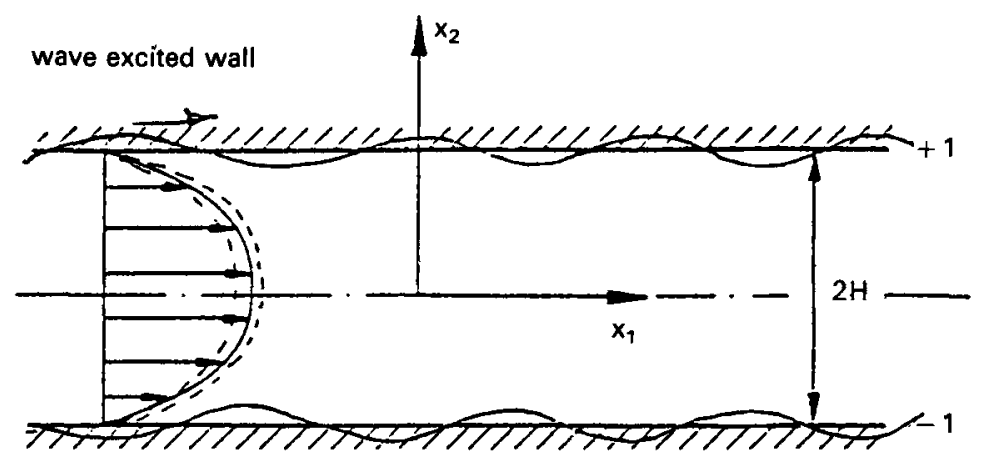

Figure 1. Flow configuration.

which represents a wave travelling in the wall plane around the position $x_{2}= \pm 1$ with amplitude $\varepsilon_{w}$, wave number vector $\left(\lambda_{1}, \lambda_{3}\right)$ and frequency $\omega_{g}$. In the above form the waves on the upper and lower walls are in phase so that at any instant of time the local channel width expands and contracts around the value $2 H$. It is relatively straightforward in principle to extend the study to permit the waves on the two walls to be out of phase with each other. This might be important for the behaviour of further disturbances and hence from the point of view of transition control. However, an additional parameter, viz. the phase difference, would then enter the picture. In the present state of such studies it is desirable to keep the number of additional parameters within limits and therefore we restrict ourselves in this work to walls excited in phase as in (2).

For small amplitudes of the wave excitation at the wall, the solution for the flow quantities may be sought as a perturbation from the parabolic velocity profile of the fully developed channel flow. To a linear approximation in $\varepsilon_{w}$ we may then write for the flow quantities:

$$
\begin{aligned}
u_{1} & =\delta_{1 l}\left(1-x_{2}^{2}\right)+\varepsilon_{w} \tilde{u}_{1}+O\left(\varepsilon_{w}^{2}\right), \quad l=1,2,3, \\
p & =-\left(2 x_{1} / \operatorname{Re}\right)+\varepsilon_{w} \tilde{p}+O\left(\varepsilon_{w}^{2}\right) .
\end{aligned}
$$

Transferring the boundary conditions in (2) to the mean position of the wall by standard methods (see, e.g. Van Dyke 1975) we get the boundary conditions for the perturbation $\tilde{u}_{1}$ as follows:

$$
\begin{aligned}
& \tilde{u}_{1}( \pm 1)=-2 \operatorname{Real}\left(\exp \left[i\left(\lambda_{1} x_{1}+\lambda_{3} x_{3}-\omega_{g} t\right)\right]\right), \\
& \tilde{u}_{2}( \pm 1)=\operatorname{Real}\left( \pm i \omega_{g} \exp \left[i\left(\lambda_{1} x_{1}+\lambda_{3} x_{3}-\omega_{g} t\right)\right]\right), \\
& \tilde{u}_{3}( \pm 1)=0 .
\end{aligned}
$$

The form of the solution for $\tilde{u}_{1}$ may then be written as

$$
\begin{gathered}
\tilde{u}_{l}=\frac{1}{2}\left[\hat{u}_{l}\left(x_{2}\right) \exp \left[i\left(\lambda_{1} x_{1}+\lambda_{3} x_{3}-\omega_{g} t\right)\right]+\right. \\
\left.\hat{u}_{t}^{*}\left(x_{2}\right) \exp \left[-i\left(\lambda_{1} x_{1}+\lambda_{3} x_{3}-\omega_{g} t\right)\right]\right], \quad l=1,2,3, \quad \\
\tilde{p}=\frac{1}{2}\left[\hat{p}\left(x_{2}\right) \exp \left[i\left(\lambda_{1} x_{1}+\lambda_{3} x_{3}-\omega_{g} t\right)\right]+\hat{p}^{*}\left(x_{2}\right) \exp \left[i\left(\lambda_{1} x_{1}+\lambda_{3} x_{3}-\omega_{g} t\right)\right]\right]
\end{gathered}
$$

where the superscript * denotes the complex conjugate. 
The complex amplitude functions for the velocity $u_{1}$ and pressure $\hat{p}$ in (5) then obey the following linearised equations of motion:

$$
\begin{aligned}
& i \lambda_{1} \hat{u}_{1}+\left(\mathrm{d} \hat{u}_{2} / \mathrm{d} x_{2}\right)+i \lambda_{3} \hat{u}_{3}=0, \\
& i\left[-\omega_{g}+\left(1-x_{2}^{2}\right) \lambda_{1}\right] \hat{u}_{1}-2 x_{2} \hat{u}_{2}= \\
& \quad-i \lambda_{1} \hat{p}+(1 / \operatorname{Re})\left[\left(-\lambda_{1}^{2}-\lambda_{3}^{2}\right)+\left(\mathrm{d}^{2} / \mathrm{d} x_{2}^{2}\right)\right] \hat{u}_{1}, \\
& i\left[-\omega_{g}+\left(1-x_{2}^{2}\right) \lambda_{1}\right] \hat{u}_{2}=-\left(\mathrm{d} \hat{p} / \mathrm{d} x_{2}\right)+(1 / \operatorname{Re})\left[\left(-\lambda_{1}^{2}-\lambda_{3}^{2}\right)+\left(\mathrm{d}^{2} / \mathrm{d} x_{2}^{2}\right)\right] \hat{u}_{2}, \\
& i\left[-\omega_{g}+\left(1-x_{2}^{2}\right) \lambda_{1}\right] \hat{u}_{3}=-i \lambda_{3} \hat{p}+(1 / \operatorname{Re})\left[\left(-\lambda_{1}^{2}-\lambda_{3}^{2}\right)+\left(\mathrm{d}^{2} / \mathrm{d} x_{2}^{2}\right)\right] \hat{u}_{3} .
\end{aligned}
$$

The above equations $(6 a-d)$ are the linearised equations of motion from which the well-known Orr-Sommerfeld equations for investigations of the more classical studies on fluid flow stability are derived. The difference from the classical problem here is that in the present case they satisfy inhomogeneous boundary conditions due to wall excitation. These are:

$$
\hat{u}_{1}( \pm 1)=-2 ; \quad \hat{u}_{2}( \pm 1)= \pm i \omega_{g} ; \quad \hat{u}_{3}( \pm 1)=0 .
$$

The set of equations (6) with the boundary conditions (7) have been solved numerically by two methods. One of the methods uses superposition coupled with an orthonormalization procedure with a variable-step Runge-Kutta-Fehlberg integration scheme (Scott \& Watts 1977), and the other is the pseudo-spectral collocation method described in Gottlieb et al (1984, pp. 1-54). The solution procedures are outlined in Selvarajan \& Vasanta Ram (1991). Plots of the velocity components for a set of parameters are shown in figures $2-7$. The frequency $\omega_{g}$ prescribed here is the same as the frequency of the neutrally stable Tollmien-Schlichting wave at the chosen wave number $\left(\lambda_{1}, \lambda_{3}\right)$.

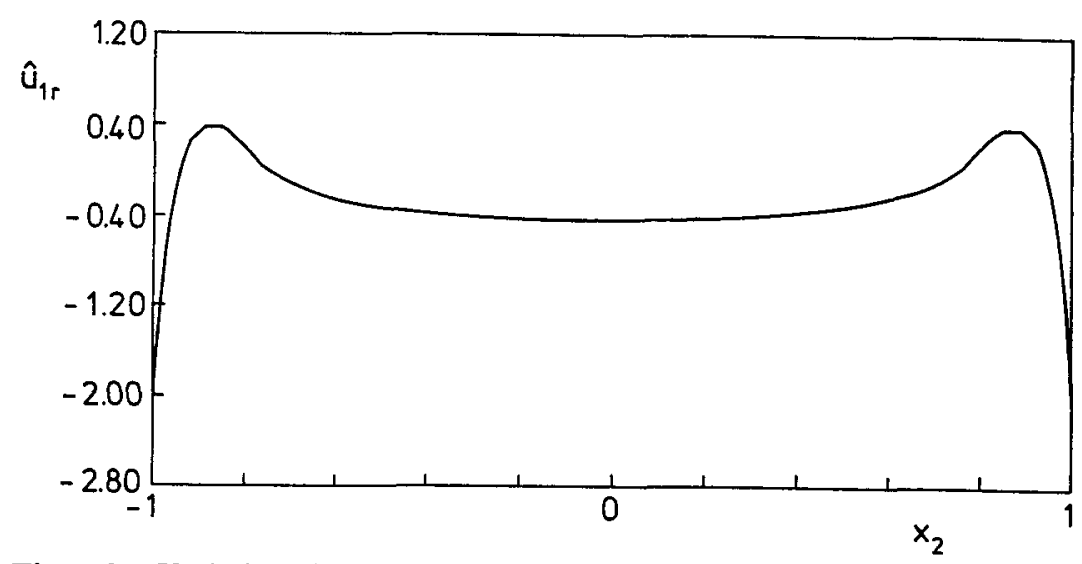

Figure 2. Variation of $\hat{u}_{1 r}$. Basic flow parameters: $\lambda_{1}=1 \cdot 04 ; \lambda_{3}=0 \cdot 29 ; \omega_{g}=0.27$. 


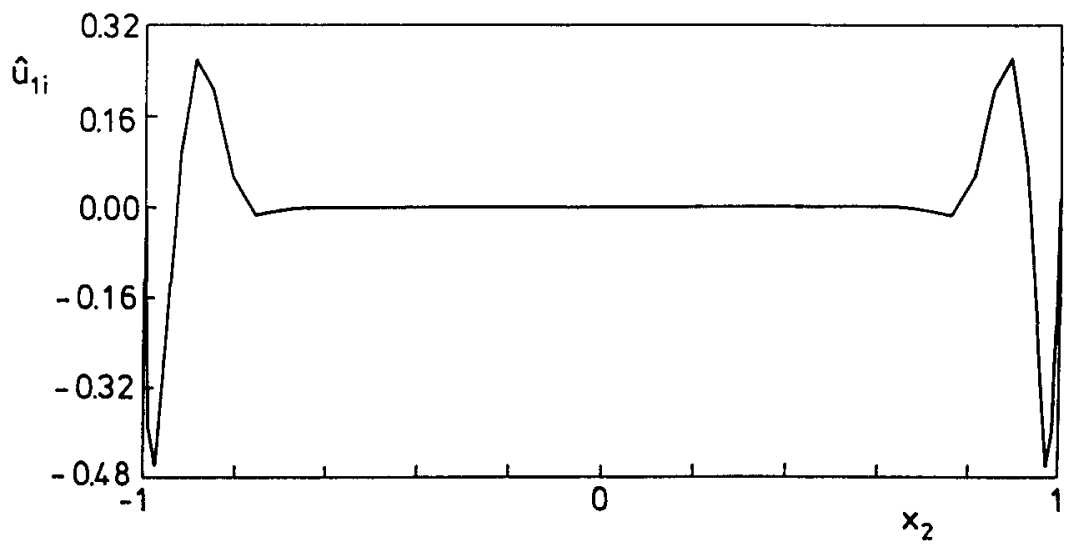

Figure 3. Variation of $\hat{u}_{1 i}$. Basic flow parameters: $\lambda_{1}=1 \cdot 04 ; \lambda_{3}=0.29 ; \omega_{g}=0.27$.

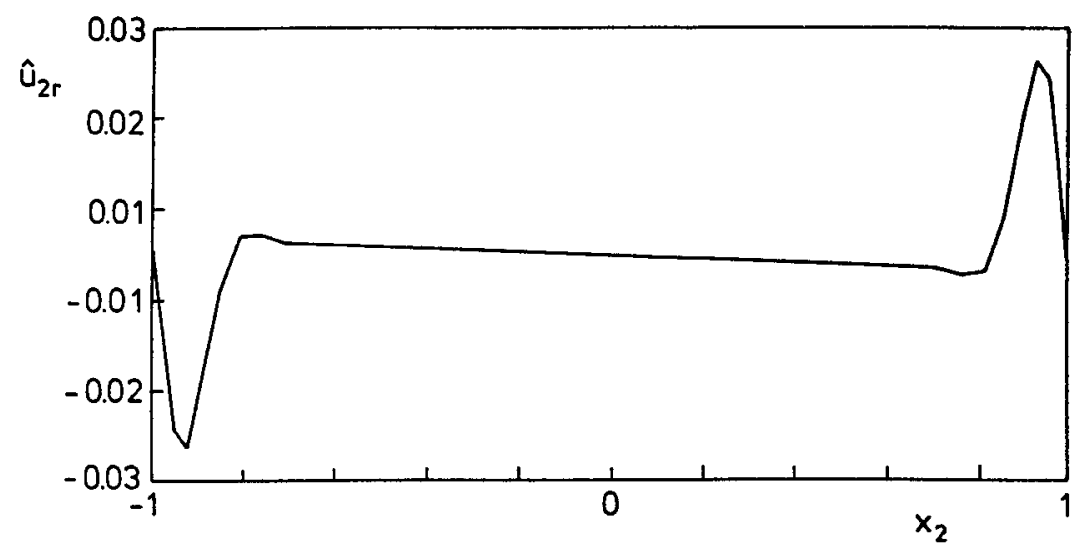

Figure 4. Variation of $\hat{u}_{2 r}$. Basic flow parameters: $\lambda_{1}=1.04 ; \lambda_{3}=0.29 ; \omega_{g}=0.27$.

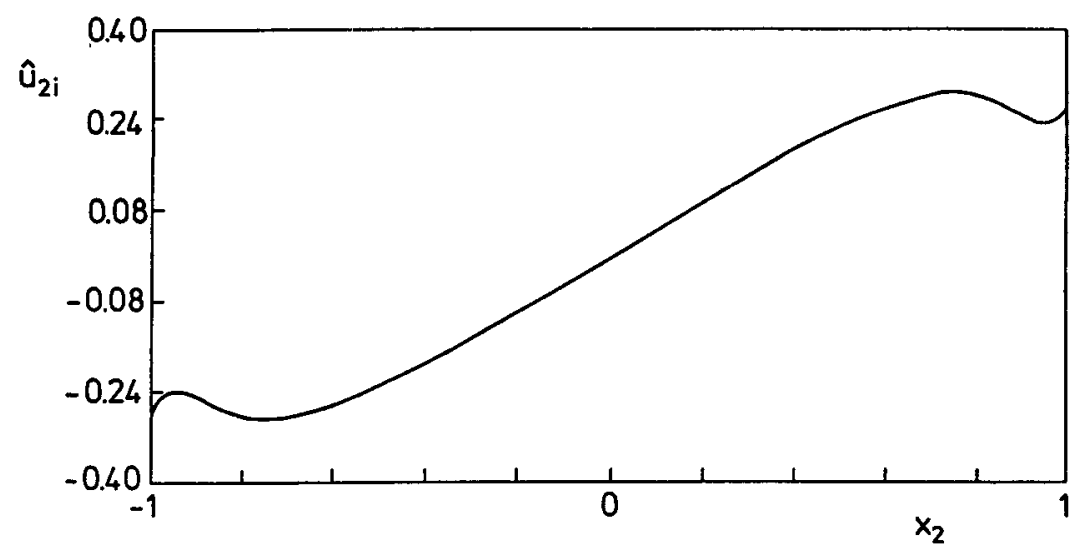

Figure 5. Variation of $\hat{u}_{2 i}$. Basic flow parameters: $\lambda_{1}=1 \cdot 04 ; \lambda_{3}=0 \cdot 29 ; \omega_{g}=0 \cdot 27$. 


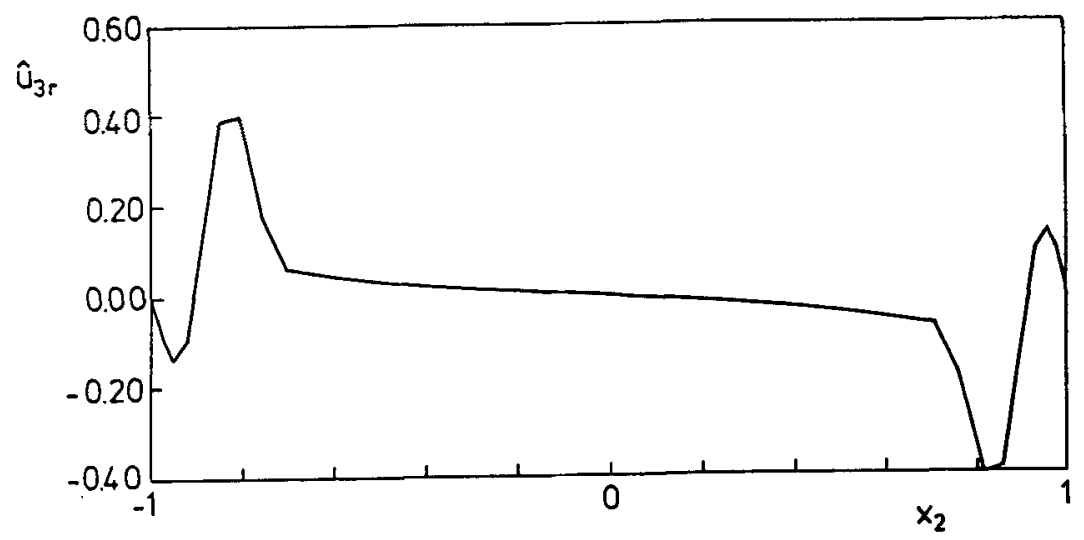

Figure 6. Variation of $\hat{u}_{3 r}$. Basic flow parameters: $\lambda_{1}=1 \cdot 04 ; \lambda_{3}=0.29 ; \omega_{g}=0.27$.

\section{The propagation of disturbances in the basic flow}

The basic flow in which we study disturbance propagation in this work is defined through (3) above. Since this contains components that are periodic in time and in two space variables, the disturbance propagation problem has to be approached through the Floquet theory. For small amplitude, further disturbances of the order $O\left(\varepsilon_{s}\right)$ over the basic flow, with $\varepsilon_{s} \ll_{w}$, we may linearise the equations of motion to obtain the governing equations for the disturbance.

Writing the velocity and pressure as

$$
\begin{aligned}
& u_{l}=\delta_{11}\left(1-x_{2}^{2}\right)+\varepsilon_{w} \tilde{u}_{l}+\varepsilon_{s} u_{s l}, \quad l=1,2,3, \\
& p=-\left(2 x_{1} / \operatorname{Re}\right)+\varepsilon_{w} \tilde{p}+\varepsilon_{s} p_{s},
\end{aligned}
$$

the linearised equations of motion for the further disturbance are

$$
\partial u_{s l} / \partial x_{1}=0
$$

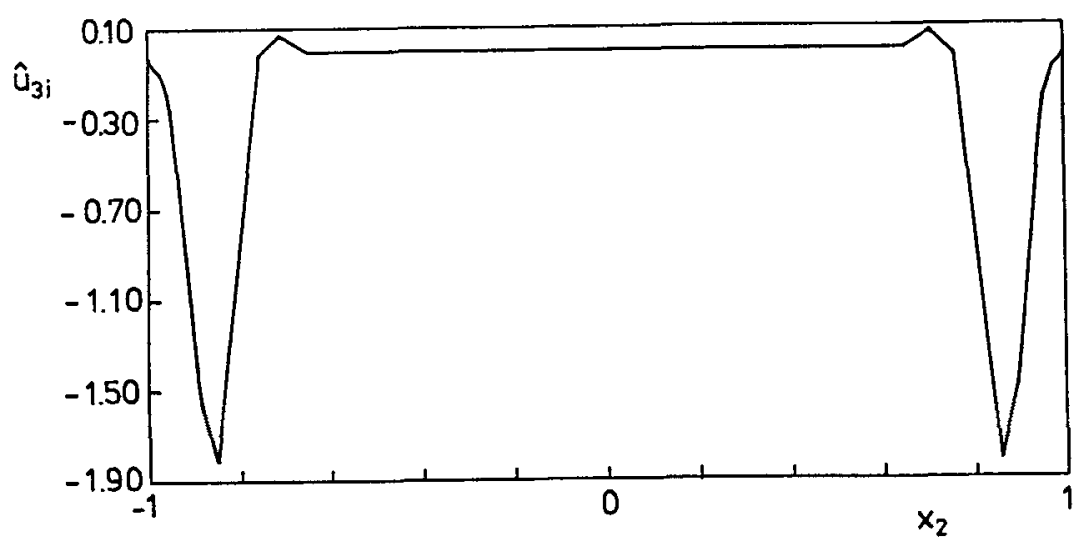

Figure 7. Variation of $\hat{u}_{3 i}$. Basic flow parameters: $\lambda_{1}=1 \cdot 04 ; \lambda_{3}=0 \cdot 29 ; \omega_{g}=0.27$. 


$$
\begin{aligned}
& \frac{\partial u_{s 1}}{\partial t}+\left(1-x_{2}^{2}\right) \frac{\partial u_{s 1}}{\partial x_{1}}-2 x_{2} u_{s 2}+\frac{\partial p_{s}}{\partial x_{1}}+\varepsilon_{w}\left[\tilde{u}_{m} \frac{\partial u_{s 1}}{\partial x_{m}}+\right.\left.u_{s m} \frac{\partial \tilde{u}_{1}}{\partial x_{m}}\right] \\
&+\frac{1}{\operatorname{Re}} \frac{\partial^{2} u_{s 1}}{\partial x_{m} \partial x_{m}}=0 \\
& \frac{\partial u_{s 2}}{\partial t}+\left(1-x_{2}^{2}\right) \frac{\partial u_{s 2}}{\partial x_{1}}+\frac{\partial p_{s}}{\partial x_{2}}+\varepsilon_{w}\left[\tilde{u}_{m} \frac{\partial u_{s 2}}{\partial x_{m}}+u_{s m} \frac{\partial \tilde{u}_{2}}{\partial x_{m}}\right]+\frac{1}{\operatorname{Re} \frac{\partial^{2} u_{s 2}}{\partial x_{m} \partial x_{m}}=0} \\
& \frac{\partial u_{s 3}}{\partial t}+\left(1-x_{2}^{2}\right) \frac{\partial u_{s 3}}{\partial x_{1}}+\frac{\partial p_{s}}{\partial x_{3}}+\varepsilon_{w}\left[\tilde{u}_{m} \frac{\partial u_{s 3}}{\partial x_{m}}+u_{s m} \frac{\partial \tilde{u}_{3}}{\partial x_{m}}\right]+\frac{1}{\operatorname{Re}} \frac{\partial^{2} u_{s 3}}{\partial x_{m} \partial x_{m}}=0
\end{aligned}
$$

Our interest lies in the stability characteristics of the wave excited basic flow due to further disturbance, so homogeneous boundary conditions for $u_{s t}$ are appropriate. It is consistent with the approximations to impose these conditions at the mean position of the channel walls $x_{2}= \pm 1$.

\section{Form of solution for the eigenvalue problem (Floquet-Ansatz)}

The outstanding feature of the disturbance equation (9) that demarcates it from more classical stability problems is the presence of periodic terms in $t, x_{1}$ and $x_{3}$ through $\tilde{u}_{1}$. It is known (see e.g. Bender \& Orszag 1984) that, although the parameter $\varepsilon_{w}$ may be small, the structure of the solution is drastically changed by the peribdic terms. The form of the solution for (9) that transforms the partial differential equation into an ordinary differential equation may be written as follows:

$$
\begin{aligned}
& u_{s l}=\sum_{n=-\infty}^{n=+\infty} A_{s l}^{(n)}\left(x_{2}\right) \exp \left[i \varphi_{s}^{(n)}\right], \quad l=1,2,3, \\
& p_{s}=\sum_{n=-\infty}^{n=+\infty} A_{s p}^{(n)}\left(x_{2}\right) \exp \left[i \varphi_{s}^{(n)}\right],
\end{aligned}
$$

where

$$
\varphi_{s}^{(n)}=\left(\alpha_{s}+n \lambda_{1}\right) x_{1}+\left(\beta_{s}+\dot{n} \lambda_{3}\right) x_{3}-\left(\omega_{s}+n \omega_{g}\right) t .
$$

The expression in (10) represents a superposition of waves of wave number $\left(\alpha_{s}+n \lambda_{1}\right)$, $\left(\beta_{s}+n \lambda_{3}\right)$ and frequency $\left(\omega_{s}+n \omega_{g}\right)$. In the temporal problem $\alpha_{s}$ and $\beta_{s}$ are regarded real. Solution of the problem then yields the complex frequency $\omega_{s}$ for which (9) admits nontrivial solutions. The real part of $\omega_{s}$ then indicates the growth or decay of the further disturbance characterised by $\left(\alpha_{s}, \beta_{s}\right)$. We write the dispersion relation of the problem symbolically in the following form

$$
F\left(\omega_{s}, \alpha_{s}, \beta_{s}, \operatorname{Re}, \varepsilon_{w}, \lambda_{1}, \lambda_{3}, \omega_{g}\right)=0,
$$

that shows the additional parameters that enter the problem due to the wave excitation of the wall. These are $\varepsilon_{w}, \lambda_{1}, \lambda_{3}$ and $\omega_{g}$.

The equations governing the eigenfunctions $A_{s l}^{(n)}\left(x_{2}\right)$ and $A_{s p}^{(n)}\left(x_{2}\right)$ are as follows,

$$
i\left(\alpha_{s}+n \lambda_{1}\right) A_{s l}^{(n)}+\frac{\mathrm{d} A_{s 2}^{(n)}}{\mathrm{d} x_{2}}+i\left(\beta_{s}+n \lambda_{3}\right) A_{s 3}^{(n)}=0
$$




$$
\begin{gathered}
i\left[-\left(\omega_{s}+n \omega_{g}\right)+\left(\alpha_{s}+n \lambda_{1}\right)\left(1-x_{2}^{2}\right)\right] A_{s 1}^{(n)}-2 x_{2} A_{s 2}^{(n)}+i\left(\alpha_{s}+n \lambda_{1}\right) A_{s p}^{(n)}+ \\
\frac{1}{\operatorname{Re}}\left[\left(\alpha_{s}+n \lambda_{1}\right)^{2}+\left(\beta_{s}+n \lambda_{3}\right)^{2}-\frac{\mathrm{d}^{2}}{\mathrm{~d} x_{2}^{2}}\right] A_{s 1}^{(n)}+\frac{\varepsilon_{w}}{2} \Phi_{1}^{(n)}=0 \\
i\left[-\left(\omega_{s}+n \omega_{g}\right)+\left(\alpha_{s}+n \lambda_{1}\right)\left(1-x_{2}^{2}\right)\right] A_{s 2}^{(n)}+\frac{\mathrm{d} A_{s p}^{(n)}}{\mathrm{d} x_{2}}+ \\
\frac{1}{\operatorname{Re}}\left[\left(\alpha_{s}+n \lambda_{1}\right)^{2}+\left(\beta_{s}+n \lambda_{3}\right)^{2}-\frac{\mathrm{d}^{2}}{\mathrm{~d} x_{2}^{2}}\right] A_{s 2}^{(n)}+\frac{\varepsilon_{w}}{2} \Phi_{2}^{(n)}=0 \\
i\left[-\left(\omega_{s}+n \omega_{g}\right)+\left(\alpha_{s}+n \lambda_{1}\right)\left(1-x_{2}^{2}\right)\right] A_{s 3}^{(n)}+i\left(\beta_{s}+n \lambda_{3}\right) A_{s p}^{(n)}+ \\
\frac{1}{\operatorname{Re}}\left[\left(\alpha_{s}+n \lambda_{1}\right)^{2}+\left(\beta_{s}+n \lambda_{3}\right)^{2}-\frac{\mathrm{d}^{2}}{\mathrm{~d} x_{2}^{2}}\right] A_{s 3}^{(n)}+\frac{\varepsilon_{w}}{2} \Phi_{3}^{(n)}=0
\end{gathered}
$$

The quantity $\Phi_{l}^{(n)}$, with $l=1,2,3$, that multiplies $\varepsilon_{w}$ in (12) is an abbreviation for the following expression,

$$
\begin{aligned}
\Phi_{l}^{(n)}= & {\left[i \hat{u}_{1}\left[\alpha_{s}+(n-1) \lambda_{1}\right]+i \hat{u}_{3}\left[\beta_{s}(n-1) \lambda_{3}\right]+\hat{u}_{2} \frac{\mathrm{d}}{\mathrm{d} x_{2}}\right] A_{s l}^{(n-1)} } \\
& +\left[i \hat{u}_{1}^{*}\left[\alpha_{s}+(n+1) \lambda_{1}\right]+i \hat{u}_{3}^{*}\left[\beta_{s}(n+1) \lambda_{3}\right]+\hat{u}_{2}^{*} \frac{\mathrm{d}}{\mathrm{d} x_{2}}\right] A_{s l}^{(n+1)} \\
& +\left[i \lambda_{1} A_{s 1}^{(n-1)}+i \lambda_{3} A_{s 3}^{(n-1)}+A_{s 2}^{(n-1)} \frac{\mathrm{d}}{\mathrm{d} x_{2}}\right] \hat{u}_{l} \\
& +\left[-i \lambda_{1} A_{s 1}^{(n+1)}-i \lambda_{3} A_{s 3}^{(n+1)}+A_{s 2}^{(n+1)} \frac{\mathrm{d}}{\mathrm{d} x_{2}}\right] \hat{u}_{1}^{*} .
\end{aligned}
$$

To bring out the differences between studies of the stability of the flow with a wave excited wall and the classical problem more clearly, it is meaningful to rewrite the ordinary differential equations for the complex amplitude velocity functions $A_{s l}^{(n)}$ in a form in which the contribution of the Orr-Sommerfeld part and of the additions can be more easily recognised. We therefore subject (12) to the following steps:

Step 1. Multiplication and addition: $\left(\alpha_{s}+n \lambda_{1}\right)+\left(\beta_{s}+n \lambda_{3}\right)$.

$$
(12 b)
$$

Step 2. $\left(\mathrm{d} / \mathrm{d} x_{2}\right)$ (outcome of step 1$)-i\left[\left(\alpha_{s}+n \lambda_{1}\right)^{2}+\left(\beta_{s}+n \lambda_{3}\right)^{2}\right]$.

Step 3. Multiplication and subtraction: $\left(\beta_{s}+n \lambda_{3}\right)-\left(\alpha_{s}+n \lambda_{1}\right)$.

The resulting equation after steps 1 and 2 above is (15) below where the abbreviation $k_{n}$ stands for

$$
\begin{aligned}
& k_{n}^{2}=\left(\alpha_{s}+n \lambda_{1}\right)^{2}+\left(\beta_{s}+n \lambda_{3}\right)^{2} \\
& \frac{\mathrm{d}}{\mathrm{d} x_{2}}\left[\left[\left(\omega_{s}+n \omega_{g}\right)-\left(\alpha_{s}+n \lambda_{1}\right)\left(1-x_{2}^{2}\right)\right] \frac{\mathrm{d} A_{s 2}^{(n)}}{\mathrm{d} x_{2}}\right]-\left(\alpha_{s}+n \lambda_{1}\right) \frac{\mathrm{d}}{\mathrm{d} x_{2}}\left[2 x_{2} A_{s 2}^{(n)}\right]+
\end{aligned}
$$




$$
\begin{aligned}
k_{n}^{2}\left[-\left(\omega_{s}+n \omega_{g}\right)+\right. & \left.\left(\alpha_{s}+n \lambda_{1}\right)\left(1-x_{2}^{2}\right)\right] A_{s 2}^{(n)}+ \\
& \frac{1}{\operatorname{Re}}\left[2 i k_{n}^{2} \frac{\mathrm{d}^{2}}{\mathrm{~d} x_{2}^{2}}-i \frac{\mathrm{d}^{4}}{\mathrm{~d} x_{2}^{4}}-i k_{n}^{4}\right] A_{s 2}^{(n)}+\frac{\varepsilon_{w}}{2} \Psi^{(n)}=0 .
\end{aligned}
$$

The quantity $\Psi^{(n)}$ which is the multiplier of $\varepsilon_{w}$ in (15) is a sum of thirteen terms as follows.

$$
\Psi^{(n)}=\sum_{j=1}^{13} \Psi_{j}^{(n)} .
$$

The $\Psi_{j}^{(n)}$ 's, $j=1,2,3, \ldots, 13$ are listed in Seivarajan \& Vasanta Ram (1991).

The outcome of step 3 is (18) below, where $\Omega^{(n)}$ stands for:

$$
\begin{aligned}
& \Omega^{(n)}=\left(\beta_{s}+n \lambda_{3}\right) A_{s 1}^{(n)}-\left(\alpha_{s}+n \lambda_{1}\right) A_{s 3}^{(n)} \\
& \begin{aligned}
i\left[-\left[\omega_{s}+n \omega_{g}\right]+\left[\alpha_{s}+n \lambda_{1}\right](1\right. & \left.\left.-x_{2}^{2}\right)\right] \Omega^{(n)}-2 x_{2}\left(\beta_{s}+n \lambda_{3}\right) A_{s 2}^{(n)} \\
& +\frac{1}{\operatorname{Re}}\left[k_{n}^{2} \Omega^{(n)}-\frac{\delta^{2} \Omega^{(n)}}{\mathrm{d} x_{2}^{2}}\right]+\frac{\varepsilon_{w}}{2} \chi^{(n)}=0 .
\end{aligned}
\end{aligned}
$$

The quantity $\chi^{(n)}$, the multiplier of $\varepsilon_{w}$ in (18), is a sum of twelve terms as follows,

$$
\chi^{(n)}=\sum_{j=1}^{12} \chi_{j}^{(n)} .
$$

The $\chi_{j}^{(n)}, j=1,2,3, \ldots, 12$, are listed in Selvarajan \& Vasanta Ram (1991).

Equations (15) and (18), together with the continuity equation (12a), are the set of equations for the complex amplitude functions of the velocity $A_{s 1}^{(n)}, A_{s 2}^{(n)}$ and $A_{s 3}^{(n)}$. The boundary condition on all these quantities is zero at $x_{2}= \pm 1$ so that the problem reduces to an eigenvalue problem requiring solution of the dispersion relation (11) for the (complex) frequency $\omega_{s}$ (temporal stability problem!).

A cursory inspection of the governing equations shows that for the case of the unexcited wall, $\varepsilon_{w}=0,(15)$ reduces to the Orr-Sommerfeld equation for $A_{s 2}^{(n)}$ and (18) to the Squire equation for $\Omega^{(n)}$. For $\varepsilon_{w} \neq 0,(12 a),(15)$ and (18) form an infinite set of coupled equations. We truncate them at $n=+1$ and -1 , setting $A_{s l}^{(n)}$ to zero for all $n>1$ and $n>-1$. We then have nine unknowns, viz. $A_{s 1}^{(-1)}, A_{s 2}^{(-1)}, A_{s 3}^{(-1)}, A_{s 1}^{(0)}, A_{s 2}^{(0)}$, $A_{s 3}^{(0)}, A_{s 1}^{(+1)}, A_{s 2}^{(+1)}$ and $A_{s 3}^{(+1)}$. The nine equations are obtainable by writing (12a), (15) and (18) for $n=-1,0$ and +1 , setting $A_{s l}^{(n)}$ to zero for all $n>1$ and $n<-1$.

\section{Outline of the solution procedure}

The solution procedure, both for the basic flow that involves inhomogeneous boundary conditions, and for the problem of further disturbances which is treated as a temporal eigenvalue problem, is given in some detail in Selvarajan \& Vasanta Ram (1991). Here we restrict ourselves to observing that the occurrence of the OrrSommerfeld operator in both the problems may be used to advantage in the computational procedures. Whereas the basic flow problem was solved by two methods (vide $\$ 2$ ) we used only the spectral collocation method together with an iterative scheme to solve the temporal eigenvalue problem. 


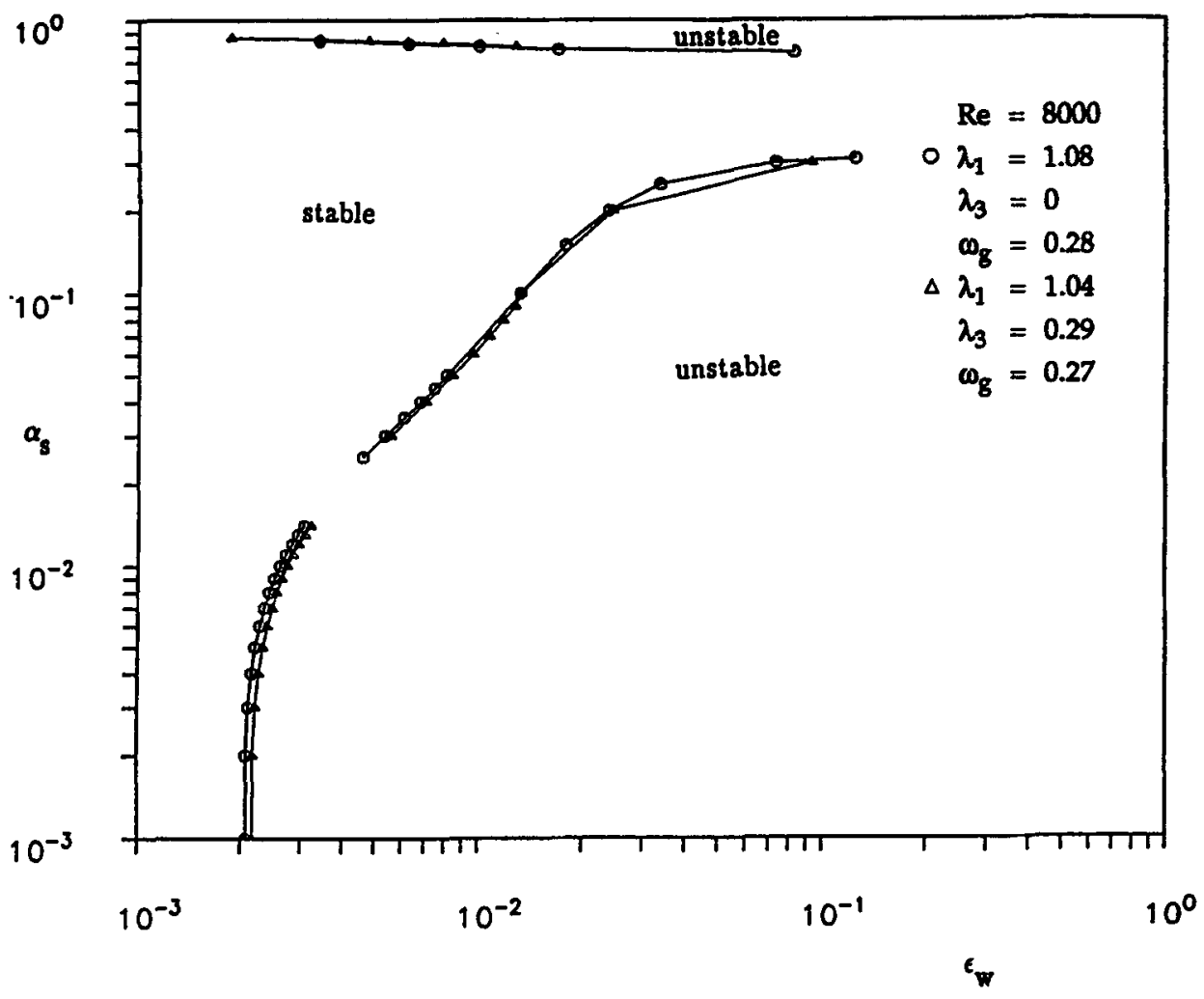

Figure 8. Stability characteristics of the wave-excited channel flow.

\section{Results}

Figure 8 shows the characteristics of the further disturbance in the $\alpha_{s}$ and $\varepsilon_{w}$ plane for a Reynolds number $\operatorname{Re}=\mathbf{8 0 0 0}$ for two sets of the other parameters whose values are indicated on the figure. Judging from our present results, which should be regarded as preliminary, there are threshold values of the wall excitation amplitude parameter $\varepsilon_{w}$ which divides the stable from the unstable regions. However, it is interesting to note that there may be disturbances at discrete bands of wave numbers which are damped even at higher amplitudes of excitation. More extensive analytical and computational studies are necessary to substantiate these findings.

A major part of this work was done when one of the authors (SS) spent a period of study at the Ruhr Universität Bochum, Germany. SS wishes to thank the Council of Scientific \& Industrial Research for leave, the German Academic Exchange Service for the award of a scholarship and Professor K Gersten, Director of the Institut für Thermo- und Fluiddynamik of the Ruhr University, Bochum for hospitality during this period. 


\section{List of symbols}

$A_{s l}^{(n)} \quad$ amplitude function of velocity disturbance, complex quantity, see (10);

$A_{s p}^{(n)} \quad$ amplitude function of pressure disturbance, complex quantity, see (10);

$F \quad$ dispersion relation, defined in (11);

$H$ mean semi-channel height;

$k_{n} \quad$ defined in (14);

(n) mode number, see (10);

p pressure;

$\tilde{p} \quad$ periodic part of pressure, see (3);

$p \quad$ amplitude function of pressure, complex quantity, see (5);

$p_{s} \quad$ pressure disturbance, defined in $\S 3, l=1,2,3$, see (8);

$\operatorname{Re} \quad$ Reynolds number, $\operatorname{Re}=U_{0} H / v$;

$t$ time coordinate;

$u_{l} \quad$ velocity component in direction $x_{l}, l=1,2,3$;

$\tilde{u}_{l} \quad$ periodic part of velocity component in $x_{l}$-direction, $l=12,3$, see (3);

$\hat{u}_{l} \quad$ amplitude function of velocity $\tilde{u}_{l}$, complex quantity, see (5);

$u_{s l} \quad$ velocity disturbance, defined in $\S 3, l=1,2,3$, see (8);

$U_{0} \quad$ mean centre line velocity;

$x_{l} \quad$ spatial coordinates, $l=1,2,3$;

$y_{w} \quad$ wall motion, defined in (2);

$\alpha_{s} \quad$ wave number of disturbance in $x_{1}$-direction, see (10c);

$\beta_{s} \quad$ wave number of disturbance in $x_{3}$-direction, see (10c);

$\varepsilon_{s} \quad$ amplitude parameter of disturbance, defined in $\S 3$, see (8);

$\varepsilon_{w} \quad$ amplitude parameter of wall motion, see (2);

$\lambda_{1} \quad$ wave number in $x_{1}$-direction, see (2);

$\lambda_{3} \quad$ wave number in $x_{3}$-direction, see (2);

$v \quad$ kinematic viscosity;

$\Phi_{l} \quad$ function defined in (13), complex quantity, $l=1,2,3$;

$\chi^{(n)} \quad$ function defined in (19);

$\Psi^{(n)} \quad$ function defined in (15);

$\omega_{g} \quad$ frequency, real quantity, see (2);

$\omega_{s} \quad$ frequency of disturbance, complex quantity, see (10c);

$\Omega^{(n)} \quad$ function defined in (17);

$l, i, j \quad$ indices;

* as superscript, denotes complex conjugate;

- above a particular symbol denotes differentiation of that parameter with respect to $x_{2}$.

\section{References}

Bender C M, Orszag S A 1984 Advanced mathematical methods for scientists and engineers (New York: McGraw Hill)

Gottlieb D, Hussaini M Y, Orszag S A 1984 Theory and application of spectral methods. In Spectral methods for partial differential equations (eds.) D Gottlieb, M Y Hussaini, S A Orszag (New York: SIAM)

Liepmann H W, Narasimha R (eds.) 1988 Turbulence management and relaminarization. IUTAM Symposium, Bangalore, India 1987 (Berlin: Springer Verlag) 
Selvarajan S, Vasanta Ram V 1991 Dynamical characteristics of wave excited channel flows. Report 162 of the Institut für Thermo- und Fluiddynamik, Ruhr Universität, Bochum Scott M R, Watts H A 1977 Computational solution of linear two-point boundary value problems via orthonormalization. SIAM J. Numer. Anal. 14: 40-70

Van Dyke M 1975 Perturbation methods in fluid mechanics (Stanford, CA: Parabolic Press) 